\section{Ligand-induced endocytosis of the pattern recognition receptor FLS2 in Arabidopsis}

\author{
Silke Robatzek, ${ }^{1,2}$ Delphine Chinchilla, and \\ Thomas Boller \\ Zurich-Basel Plant Science Center, Botanical Institute, \\ University of Basel, 4056 Basel, Switzerland
}

\begin{abstract}
Pattern-recognition receptors (PRRs) trigger innate immune responses in animals and plants. One such PRR is the flagellin receptor FLS2 in Arabidopsis. Here, we demonstrate that a functional fusion of FLS2 to the green fluorescent protein (GFP) resides in cell membranes of most tissues. Stimulation with the flagellin epitope flg22 induces its transfer into intracellular mobile vesicles, followed by degradation. FLS2 internalization depends on cytoskeleton and proteasome functions, and receptor activation. A variant FLS2 mutated in Thr 867, a potential phosphorylation site, binds flg22 normally, but is impaired in flg22 responses and FLS2 endocytosis. We propose that plant cells regulate pathogen-associated molecular pattern (PAMP)-mediated PRR activities by subcellular compartmentalization.
\end{abstract}

Supplemental material is available at http://www.genesdev.org.

Received September 19, 2005; revised version accepted January 11, 2006.

A key aspect of the eukaryotic innate immune system is the recognition of pathogen-associated molecular patterns (PAMPs), universally conserved patterns in whole classes of microbes, mediated by germline encoded, nonrearranging receptors, the so-called pattern recognition receptors (PRRs) (Gordon 2002). In vertebrates, the most important PRRs are the Toll-like receptors (TLRs), of which 11 have been identified to date. PAMP recognition through TLRs induces a conserved host defense response including production of inflammatory cytokines and antimicrobials. Furthermore, TLR signaling influences phagocytosis and triggers adaptive immunity (Pasare and Medzhitov 2004; Underhill and Ganter 2004). Mammalian TLR5 influences host susceptibility to infection through recognition of the bacterial flagellin protein, the main subunit building the flagellar structure (Smith et al. 2003).

In plants, the paradigm for a PRR is the well-characterized FLS2 receptor kinase (Gomez-Gomez et al. 2001; Asai et al. 2002; Zipfel et al. 2004). Arabidopsis FLS2 mediates flagellin recognition on the basis of the highly conserved flg22 peptide (Gomez-Gomez et al. 2001; Chinchilla et al. 2006). Interestingly, the epitope recog-

[Keywords: Endocytosis; FLS2; innate immunity; PAMP; RLK] ${ }^{1}$ Present address: Max-Planck-Institut für Züchtungsforschung, Carlvon-Linné-Weg 10, 50829 Köln, Germany.

${ }^{2}$ Corresponding author.

E-MAIL robatzek@mpiz-koeln.mpg.de; FAX 49-221-5062-353. Article and publication are at http://www.genesdev.org/cgi/doi/10.1101/ gad.366506. nized by mammalian TLR5 is different (Smith et al. 2003; Chinchilla et al. 2006). Perception of flg22 by FLS2 leads almost immediately to the generation of reactive oxygen species (ROS), protein phosphorylation, mitogenactivated protein (MAP) kinase signaling, and transcriptional gene induction (Felix et al. 1999; Asai et al. 2002; Zipfel et al. 2004). Recent work has shown that flagellin perception is essential for efficient host defense, since fls2 mutant plants exhibit an enhanced disease susceptibility to bacterial infections (Zipfel et al. 2004). Besides basal immunity, plants contain a specific defense system based on the detection of pathogen-derived race-specific avirulence factors (Avr) through corresponding products of resistance (R) genes. Recently, RIN4, a key regulator of $\mathrm{R}$-gene-mediated defense, has been shown to also modulate flg22 signaling (Kim et al. 2005). Thus, both basal and specific immune signaling pathways in plants appear to be linked.

Obviously, TLR signaling needs to be kept in check by negative regulation. Several negative regulators of TLR signaling have been recently described, which function on different levels, for example, through extracellular PAMP scavengers, cytosolic adapter proteins, or transmembrane protein regulators, or by down-regulation of TLR expression (Liew et al. 2005). Although FLS2-triggered immune responses in plants are well characterized, it is not yet clear how FLS2 signaling is attenuated. FLS2 belongs to the large receptor-like kinase (RLK) family with $~ 610$ members in Arabidopsis (Shiu and Bleecker 2001). One possibility for down-regulating RLK signaling in plants is the kinase-associated protein phosphatase (KAPP), which has been identified to interact with several RLKs including FLS2 (Gomez-Gomez et al. 2001; Becraft 2002). In addition, since RLKs are structurally related to receptor tyrosine kinases (RTK) in animals (Shiu and Bleecker 2001), mechanisms established for RTKs could also take place for RLKs. Attenuation of RTK signaling can be achieved by internalization of the receptors, either constitutively or selectively upon ligand binding (Katzmann et al. 2002).

The occurrence of endocytic processes in plants has been discovered only recently, including the possibility of constitutive RLK internalization (Shah et al. 2002; Russinova et al. 2004; Gifford et al. 2005). However, nothing is known about ligand-mediated receptor endocytosis in plants. Here, we use labeling of the FLS2 receptor with the green fluorescent protein (GFP) in order to demonstrate cell membrane localization of the FLS2 receptor and its specific, ligand-induced internalization. FLS2 accumulates in intracellular vesicles that are trafficking within the cell and likely target FLS2 for degradation. We also show that a potentially phosphorylated threonine residue in the juxta membrane region of the receptor is required for both signaling and endocytosis, indicating a close relationship between the two processes.

\section{Results and Discussion}

We transformed the Arabidopsis ecotype Ws-0, an f1s2 mutant (Zipfel et al. 2004), with a construct encoding FLS2 fused to GFP with a myc-linker under the control of its native promoter (Ws-0/FLS2-GFP). Transgenic lines exhibited expression of the full-length FLS2-GFP 
fusion protein (Supplementary Fig. 1A). Transgenic FLS2-GFP and endogenous FLS2 were expressed with similar abundance (data not shown). Expression of FLS2GFP restored normal specific flg22 binding in Ws-0 (Supplementary Fig. 1B), and the whole array of full flg22 responses, as lines expressing FLS2-myc only (Supplementary Fig. 1C; data not shown). Thus, the FLS2-GFP fusion protein is biologically equivalent to native FLS2 in every respect, making it perfectly suited for localization studies.

Confocal microscopy revealed that the FLS2-GFP fusion protein was expressed in roots, rosette leaves, stems, and flower petals, and that it was localized at the cell periphery (Fig. 1). In leaves, expression occurred in mesophyll and epidermal cells as well as in the guard cells lining the stomata, the possible entry sites for invading bacteria (Fig. 1A,B). Obviously, plants are constantly ready to detect flagellin, and thus to trigger an immediate early defense response upon a bacterial invasion. In fact, it has been demonstrated that FLS2 plays a role in the first line of host defense (Zipfel et al. 2004). Plasmolysis experiments showed a retraction of the fluorescence signal associated with the cell membrane and with Hechtian strands (Fig. 1G). This is consistent with the function of FLS2 as a surface receptor recognizing an extracellular ligand, and is in agreement with its RLK structure and biochemical ligand-binding studies (Gomez-Gomez et al. 2001). Interestingly, senescent leaves did not exhibit FLS2 expression (data not shown).

We next tested the effect of the ligand flg22 on FLS2 localization. Young rosette leaves were preincubated in water for $30 \mathrm{~min}$, treated with flg22, and then subjected to confocal microscopy. Within $20-40$ min of flg22 treatment, the fluorescent signal disappeared from the cell membrane. Instead, numerous distinct fluorescently labeled vesicles occurred underneath the cell membrane,

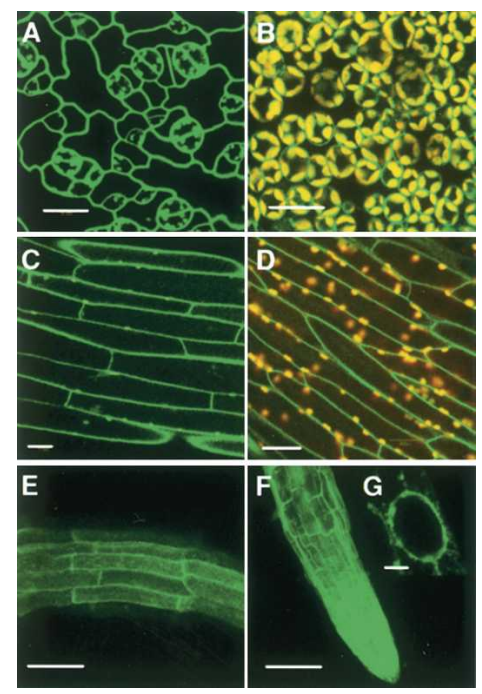

Figure 1. FLS2 expression analysis revealed by confocal fluorescence microscopy of Ws-0/FLS2-GFP lines. Micrographs show optical cross-sections $(A-D, G)$ or merged cross-sections $(E, F)$ of FLS2 cell membrane localization in different tissues. $(A)$ Leaf epidermal cells (pavement and stomata cells). (B) Leaf mesophyll cells. $(C)$ Stem. $(D)$ Flower petal. $(E)$ Root. $(F)$ Root tip. Background autofluorescence of chloroplasts appears in yellow/red. (G) Retraction of FLS2-GFP-labeled cell membrane from root cell walls upon addition of $1 \mathrm{M}$ sorbitol. Bars: $A-D, 20 \mu \mathrm{M} ; E, F, 100 \mu \mathrm{M} ; G, 10 \mu \mathrm{M}$.
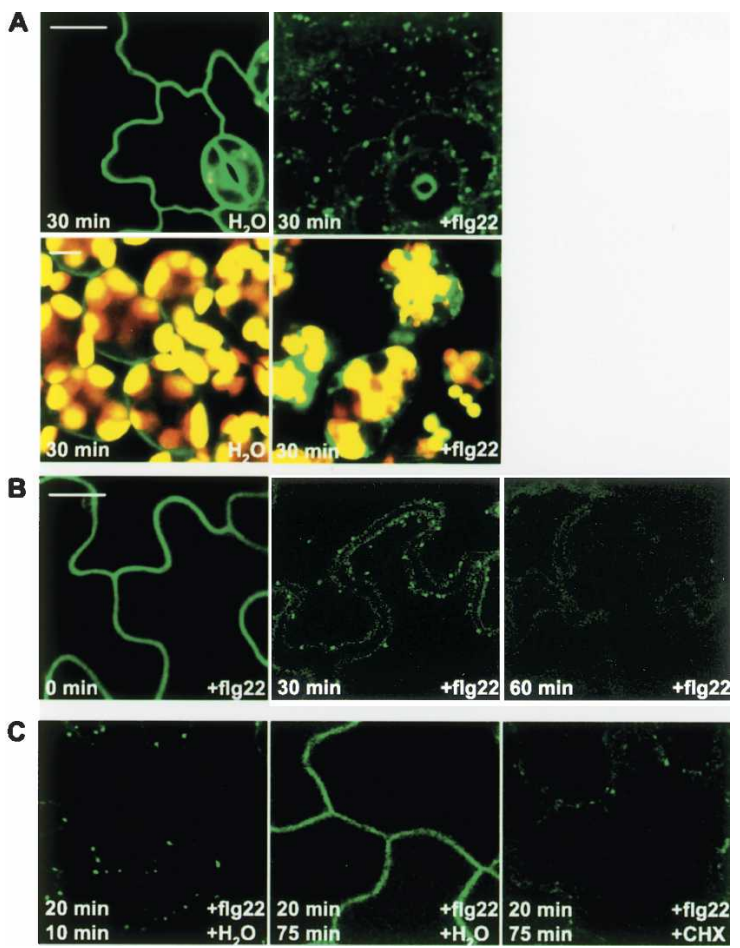

Figure 2. Dynamics of FLS2 localization in response to flg22. $(A)$ Detached leaves of Ws-0/FLS2-GFP seedlings were incubated in an aqueous solution supplied with $10 \mu \mathrm{M}$ flg22. Fluorescence micrographs show GFP signals of optical cross-sections in epidermal cells (top) and mesophyll cells (bottom) at $30 \mathrm{~min}$. Background autofluorescence of stomata inner sites stays green; background autofluorescence of chloroplasts appears in yellow/red. Leaves of Ws-0/FLS2GFP were either incubated continuously in $10 \mu \mathrm{M}$ flg22 $(B)$ or pulseincubated for $20 \mathrm{~min}$, intensively rinsed, and further incubated in water or cyclohexamide $(\mathrm{CHX})(C)$. Fluorescence micrographs show GFP signals at the indicated time points (depicted are cross-sections of epidermal cells). Bars, $10 \mu \mathrm{m}$.

both in epidermal cells and in mesophyll cells (Fig. 2A). Prolonged incubation led to a complete loss of the GFP signal, suggesting disappearance of the internalized FLS2-GFP fusion protein (Fig. 2B). Similarly, a pulse treatment with flg22 for $20 \mathrm{~min}$ followed by intense washing and a further incubation for 10 min revealed the occurrence of intracellular vesicles. However, within 75 min, the FLS2-GFP signal in the cell membrane was almost back to its original level (Fig. 2C). Thus, upon withdrawal of flg22, the cell membrane was replenished with FLS2-GFP. This replenishment was completely blocked by cycloheximide (CHX), indicating that it was based on de novo protein synthesis rather than vesicle recycling (Fig. 2C). We suggest that FLS2, upon interaction with an agonist-active ligand, is subject to degradation via endocytosis and lysosomal and/or proteasomal pathways.

Furthermore, we addressed the specificity of flg22. induced FLS2 internalization. The inactive variant flg22 $2^{\text {A.tum }}$, derived from the sequence of Agrobacterium tumefaciens flagellin (Felix et al. 1999), did not affect FLS2-GFP localization (Fig. 3). In addition, the antagonistic peptide flg22 22 (Bauer et al. 2001) failed to induce FLS2 internalization, and interestingly, a slight increase of the FLS2-GFP cell membrane signal could be observed. It is possible that the binding of an antagonist inhibits a constitutive turnover/recycling mechanism. A 


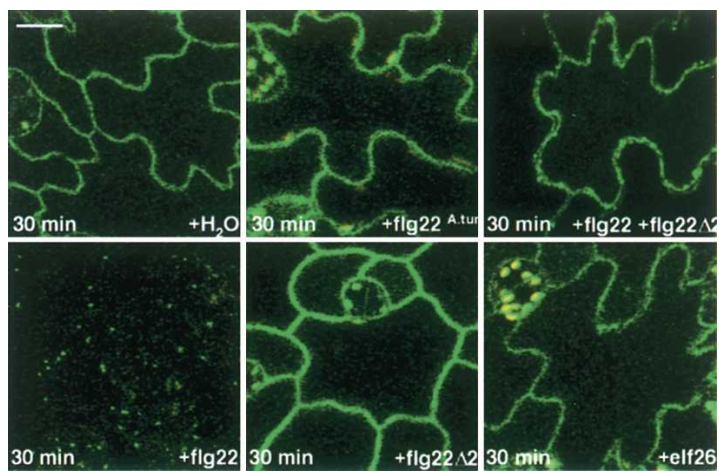

Figure 3. Specificity of FLS2 internalization. Fluorescence micrographs show optical cross-sections of GFP signals in epidermal cells of Ws-0/FLS2-GFP-derived detached leaves at $30 \mathrm{~min}$ of incubation with the elicitor active peptides flg 22 and elf 26 , the inactive variants flg $22^{\text {A.tum }}$ and flg $22 \Delta 2$, and a cotreatment with flg 22 and flg2 $2 \Delta 2$ (all at $10 \mu \mathrm{M})$. Bar, $10 \mu \mathrm{m}$. Background autofluorescence of chloroplasts appears in yellow.

simultaneous treatment of flg22 and flg22 2 at the same concentration led to fluorescence signals in cell membranes and intracellular vesicles (Fig. 3), as expected in view of the competitive behavior of the two peptides (Bauer et al. 2001). No accumulation of FLS2-GFP in vesicles was observed when leaves were incubated with the peptide elicitor elf26 (Fig. 3), a PAMP recognized by a different receptor (Kunze et al. 2004; G. Kunze, D. Chinchilla, and D. Felix, unpubl.). Since the responses to elf26 and flg22 are almost identical (Kunze et al. 2004), FLS2-GFP internalization is not due to cellular changes such as ion fluxes or the formation of ROS induced by PAMPs in general but strictly depends on binding of the ligand.

To further characterize the phenomenon of flg22-induced FLS2 internalization, we tested a series of different inhibitors. Treatment with each of the inhibitors alone did not affect FLS2 cell membrane localization (data not shown). It has been demonstrated in yeast and mammalian cells that actin plays an important role during receptor-mediated endocytosis (Engqvist-Goldstein and Drubin 2003), and we actually observed intracellular movement of the FLS2-GFP-labeled vesicles rapidly trafficking along cytoplasmic strands (Fig. 4). We found that Oryzalin, a well-known inhibitor of tubulin polymerization, and Latrunculin B, an inhibitor of actin depolymerization (Mathur and Chua 2000), strongly reduced the appearance of flg22-induced FLS2-GFP vesicles (Fig. 4). Thus, cytoskeleton components are important for FLS2 endocytosis per se, possibly through dynamin-like proteins. They function in most endocytic processes in animals and have also been found in plants (Samaj et al. 2004).

Post-Golgi-derived vesicles are known to be affected by Brefeldin A (BFA) (Emans et al. 2002). In BFA pretreated leaves, flg22 addition induced the appearance of fluorescent vesicles (Fig. 4). To further examine the nature of these vesicles, we used Wortmannin, which is thought to inhibit fusion of prevacuolar compartments (Emans et al. 2002). Application of flg22 in the presence of Wortmannin failed to induce the appearance of fluorescent vesicles (Fig. 4), indicating that these vesicles arise from a Wortmannin-sensitive endocytic process. Moreover, low temperature was also found to inhibit flg22-triggered FLS2 internalization (data not shown).
Endocytosis has been well studied in yeast and mammalian cells, while its very occurrence in plants was doubtful for many years (see Meckel et al. 2004, and references cited therein). However, during the past years, it has become clear that endocytosis takes place in plants (Samaj et al. 2004; Tse et al. 2004), even in fully turgescent guard cells (Meckel et al. 2004). It has been mainly studied in developmental processes such as cytokinesis and root hair and root tip growth, as well as stomata closing. During plant growth and development, various processes are regulated by polar transport of the phytohormone auxin, which is mediated by a polar distribution of the auxin efflux carrier PIN1 through its trafficking from endosomal compartments (Geldner et al. 2003). Auxin itself has been shown to negatively regulate PIN1 endocytosis (Paciorek et al. 2005).

Only recently, endocytosis of surface receptors has been also discovered in plants (Shah et al. 2002; Ron and Avni 2004; Russinova et al. 2004; Gifford et al. 2005). However, internalization of the RLK-type hormone receptors Brassinosteroid-Insensitive 1 (BRI1) and BRI1-associated receptor kinase (BAK1) appeared independent of ligand addition and occurred constitutively (Russinova et al. 2004). In contrast, we demonstrate here that FLS2 internalization is triggered specifically by binding of its ligand flg22 and requires receptor activation. Thus, it represents the first case of ligand-mediated receptor endocytosis in plants. Induced FLS2 endocytosis is further followed by receptor degradation. This suggests, in contrast to early types of endosomes recycling plasma membrane proteins, that activated FLS2 is eventually sorted

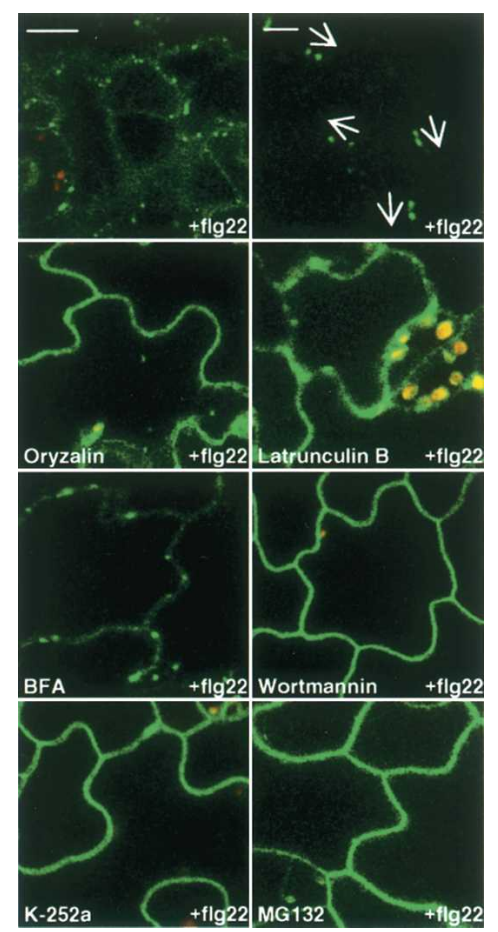

Figure 4. Regulation of FLS2 internalization. Representative fluorescence micrographs of FLS2-GFP localization in epidermal cells (cross-sections) $30 \mathrm{~min}$ pretreated with the indicated inhibitors, or cotreated with K-252a shown 30 min after the addition of $10 \mu \mathrm{M}$ flg22 in $1 \%$ DMSO. Bar, $10 \mu \mathrm{m}$. Arrows indicate movement of fluorescent vesicles in a time lapse of $3.5 \mathrm{sec}$. Bar, $5 \mu \mathrm{m}$. Background autofluorescence of chloroplasts appears in yellow/red. 
into late endosomal compartments referred to as multivesicular bodies (MVBs) (Katzmann et al. 2002). Nevertheless, a much slower constitutive form of turnover of FLS2 might occur as well, since treatment with the antagonistic peptide flg22 $\Delta 2$ slightly increased the fluorescence signal of cell-membrane-localized FLS2-GFP, indicating that this peptide, which prevents signaling of FLS2, also stabilizes it in the membrane.

Autophosphorylation has been shown to play a crucial role in animal RTK activation (Hubbard and Till 2000) and also occurs in several plant RLKs including FLS2 (Nasrallah 2000; Gomez-Gomez et al. 2001; Wang et al. 2001). In order to study the role of protein phosphorylation in receptor kinase endocytosis, we used K-252a, which acts as a general kinase inhibitor and was shown to inhibit elicitor responses (Felix et al. 1991). In the presence of K-252a, flg22-induced FLS2 endocytosis was completely abolished (Fig. 4). Thus, FLS2 internalization requires kinase activities, possibly that of its own serine/ threonine kinase (Gomez-Gomez et al. 2001). We next conducted site-directed mutagenesis of potential phosphorylation sites in the cytoplasmic part of FLS2 and assayed their function in flg22 binding and response by transformation of Ws-0 plants. Threonine and serine residues were selected for mutational analysis based on their high conservation among several RLKs. Transgenic lines expressing similar amounts of mutant FLS2 protein were chosen for further functional characterization (Supplementary Fig. 2A). Among five different threonine and serine residues tested (T867, S878, T1040, T1072, and S1118), we could identify three (FLS2 ${ }^{\text {T867V }}$, FLS2 ${ }^{\text {T1040A }}$, FLS2 ${ }^{\text {T1072A }}$ ) that abolished flg22-induced immediate generation of ROS (Supplementary Fig. 2B). Two mutations, FLS2 ${ }^{\mathrm{T} 867 \mathrm{~V}}$ and FLS2 ${ }^{\mathrm{T} 1040 \mathrm{~A}}$, also strongly affected flg22 downstream signaling, since seedling growth was not inhibited in the presence of $100 \mathrm{nM} \mathrm{flg} 22$ (Supplementary Fig. 2C). A partial seedling growth inhibition by flg22 was observed in FLS2 ${ }^{\text {S878A }}$ - and FLS2 ${ }^{\text {T1072A }}$-expressing lines. Only FLS2 ${ }^{\text {S1118A }}$ was capable of mediating a full flg22 response (Supplementary Fig. 2B,C). Unlike the described mutations f1s2-17 and f1s2-24 (Gomez-Gomez et al. 2001), all mutations, even those impaired in flg22 responses, exhibited clear flg22 binding (Supplementary Fig. 2D).

One of the identified residues, FLS2 ${ }^{\mathrm{T} 867 \mathrm{~V}}$, was located in the juxta membrane region of FLS2, a region known to be phosphorylated in RLKs (Nuhse et al. 2004; Wang et al. 2005), and to be important in RTK function by creating docking sites for recruitment of downstream molecules (Hubbard and Till 2000). Moreover, FLS2 ${ }^{\mathrm{T} 867}$ resembles BRI1 ${ }^{\mathrm{T} 880}$, which has been identified as in vivo phosphorylation site (Wang et al. 2005). Therefore, FLS2 ${ }^{\mathrm{T} 867 \mathrm{~V}}$ was further investigated in detail. Transgenic Ws-0/FLS2 ${ }^{\mathrm{T} 867 \mathrm{~V}}$ plants were found to exhibit the same flg22-binding characteristics as Ws-0/FLS2 plants (G. Kunze, unpubl.). However, these lines were flg22 insensitive at concentrations that induce a strong response in wild-type plants (Fig. 5A,B). In addition, they also displayed an enhanced disease susceptibility phenotype upon infection with pathogenic Pseudomonas syringae bacteria (Fig. 5C). Microscopic analysis of Ws-0 transgenic plants expressing a GFP-tagged version of FLS2 ${ }^{\mathrm{T} 867 \mathrm{~V}}$ showed normal cell membrane localization of the mutant FLS2 protein (Fig. 5D). Clearly, the impairments in the flg22 response of Ws-0/FLS2 ${ }^{\mathrm{T} 8} 6 \mathrm{~V} \mathrm{~V}$ plants are not a result of FLS2 ${ }^{\mathrm{T} 867 \mathrm{~V}}$ subcellular mislocalization in

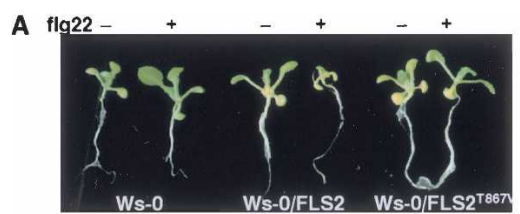

B

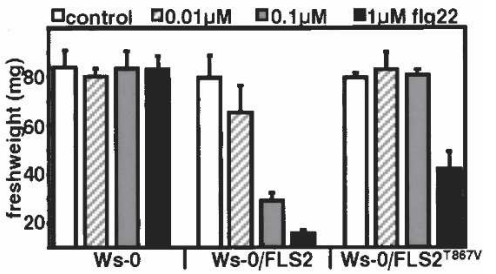

C

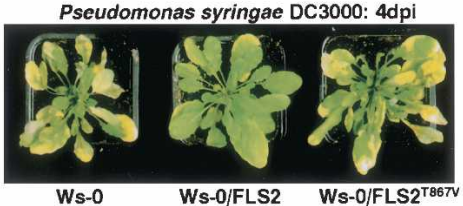

D

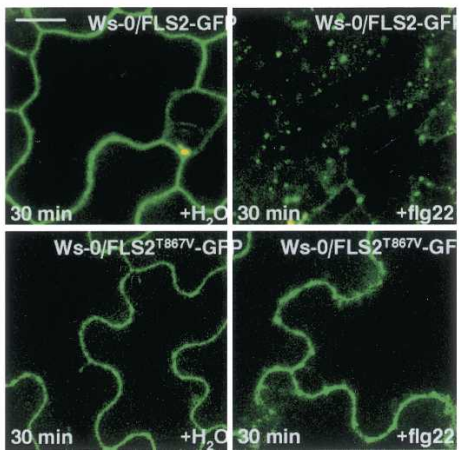

Figure 5. (A) Photographs presenting seedling growth of Ws-0 plants and lines expressing FLS2 and FLS2 $2^{\mathrm{T} 867 \mathrm{~V}}$ in the absence or presence of $0.1 \mu \mathrm{M}$ flg22. Each two independent lines are represented. $(B)$ Dose dependence of flg22-induced seedling growth inhibition in Ws-0 plants and lines expressing FLS2 and FLS2 ${ }^{\mathrm{T} 867 \mathrm{~V}} ; n=6$ seedlings of each representative line. (C) FLS2-mediated disease resistance. Ws- 0 plants and lines expressing FLS2 and FLS2 ${ }^{\text {T867V }}$ were spray-inoculated with $5 \times 10^{8} \mathrm{CFU} / \mathrm{mL}$ bacteria of Pseudomonas syringae pv. tomato DC3000. Photographs were taken $4 \mathrm{~d}$ postinoculation and each shows a representative line. Disease occurs visible by leaf yellowing. $(D)$ Subcellular localization of FLS2 ${ }^{\mathrm{T} 867 \mathrm{~V}}$. GFP expressed in Ws-0. Confocal microscopy revealed a fluorescence signal at the cell membrane that stayed mostly unchanged in the presence of $10 \mu \mathrm{M}$ flg22 (cross-section of epidermal cells). Bars, $10 \mu \mathrm{M}$.

the absence of elicitor. However, upon flg22 addition, FLS2 ${ }^{\mathrm{T} 867 \mathrm{~V}}$ endocytosis was strongly reduced (Fig. 5D), and only observed as a delayed rare event. Therefore, mutation of the threonine residue 867 hampered both FLS2 internalization and FLS2 response, indicating that both processes are intimately connected.

For surface receptor endocytosis, ligand binding induces receptor kinase activation and phosphorylational and conformational changes that lead to the exposition of sorting motifs recognized by cytosolic proteins. Classically, the tetrapeptide YXXФ functions as a sorting signal in animal receptors (Kurten 2003). Although this motif can be found in plant RLKs and PRRs (Ron and Avni 2004), it is not present in FLS2. Instead, in the FLS2 cytoplasmic part there is a well-conserved PEST-like motif, which has been implicated in ubiquitin-triggered 
receptor endocytosis (Haglund et al. 2003). Further evidence for a possible FLS2 ubiquitination comes from our gene chip expression analysis. Among flg22 rapidly induced genes are several coding for RING zinc-finger proteins with potential E3-ligase activities (Zipfel et al. 2004). E3 ligases have been reported to regulate TLRs (Chuang and Ulevitch 2004). Furthermore, ligand-induced receptor ubiquitination is important for their endocytic sorting into lysosomes and finally degradation (Longva et al. 2002). Interestingly, lysosomal receptor degradation appears to be regulated by the proteasome. Inhibition of the proteasomal activity affects the uptake of internalized receptors from outer membranes of MVBs into small inner vesicles that will fuse with the lysosome (Haglund et al. 2003). Eventually, internalized receptors can recycle back to the cell membrane (van Kerkhof and Strous 2001). Trafficking of the epidermal growth factor receptor (EGFR) to MVBs requires sorting signals within the cytoplasmic tail and also function of an ubiquitin ligase (Longva et al. 2002). Studies using proteasome inhibitors revealed that not only FLS2 degradation but also the internalization step were strongly compromised by MG132 (Fig. 4) and LNLL (data not shown). This suggests that the proteasome activity primarily influences FLS2 endocytosis and rather indirectly its degradation, as has been described for RTKs (Hammond et al. 2001). Therefore, we are currently addressing the involvement of the PEST motif in the mechanism of FLS2 endocytosis and introduced a single amino acid exchange within the FLS2 PEST-like motif. Preliminary results of Ws-0 transgenic lines expressing this FLS2 mutant variant revealed that FLS2 endocytosis was impaired (data not shown). Intriguingly, these plants were strongly compromised in their flg22 response as assayed by flg22-mediated inhibition of seedling growth. In contrast, immediate early flg22-induced ROS production appeared to be much less affected (data not shown). These data provide evidence that FLS2 endocytosis and downstream signaling might be regulated through its PESTlike motif, possibly involving ubiquitin ligase activities.

The most studied model of ligand-mediated receptor internalization is the EGF receptor in mammals. EGF binding accelerates EGFR rapid endocytosis, which is followed by sorting and trafficking through a series of endosomal compartments. EGFR directed into MVBs results in their lysosomal degradation and therefore in signal termination (Sorkin and Von Zastrow 2002). However, the rate of receptor internalization appears to be much higher than the rate of receptor degradation. This fact together with other observations provides evidence that RTKs can signal from endosomes (Sorkin and Von Zastrow 2002). Similarly, FLS2 is internalized and likely further subjected to protein degradation, which occurs much slower than its internalization, and substantial pools of initially activated FLS2 receptor accumulate inside the cell. Thus, there is a clear possibility that FLS2 can signal from intracellular compartments, for example, through interaction with MAPK cascades. Because all flg22-triggered responses display a transient behavior (Felix et al. 1999; Zipfel et al. 2004), we postulate that this characteristic could be achieved through FLS2 internalization.

Here, we show for the first time that a plant cell membrane receptor, in its function recognizing a bacterial PAMP, exhibits ligand-induced internalization. Our results with the FLS2 $2^{\mathrm{T} 867 \mathrm{~V}}$ mutant provide evidence that endocytosis and signaling function are coupled. However, whether FLS2 internalization is, in fact, required for signaling needs to be further evaluated. What is clear, instead, is that FLS2 is subject to protein degradation after endocytosis. This implies a host cell mechanism to dispose activated PRRs, thereby down-regulating signaling capacity. Attenuation of PAMP-mediated PRR signaling in animals occurs mainly through interactions with negative regulators (Liew et al. 2005). It has been reported that uncontrolled hyperinduction of proinflammatory cytokines by TLRs may play a role in the pathogenesis of autoimmune, chronic inflammatory, and infectious diseases (Cook et al. 2004). Therefore, especially during immune responses, tailoring of pathogen-induced signaling is important to ensure both, effective defense and host survival.

\section{Materials and methods}

Generation of transgenic plants

A FLS2p::FLS2-3xmyc-GFP construct was used to transform Arabidopsis thaliana Ws-0 (Ws-0/FLS2-GFP). The FLS2 promoter up to -988 base pairs was amplified by PCR and introduced into the EcoRI and HindIII sites of pCAMBIA 2300, additionally adding BamHI and $\mathrm{KpnI}$ restriction sites upstream of the HindIII site. The FLS2 gene triple myc-tag fusion was PCR-amplified, in which the stop codon was mutated and a SalI site was introduced, and cloned into the BamHI and KpnI sites of pCAMBIA. The GFP-tag was PCR-amplified and in-frame cloned into the SalI site. Constructs were verified by sequencing. Stable transgenic lines were generated using the standard $A$. tumefaciens-mediated gene transfer procedure of inflorescence infiltration. Independent transformed plant pools were kept separate for selection of independent transgenic lines based on their kanamycine resistance. Functional complementation of Ws-0 by FLS2p::FLS2-3xmyc-GFP was assayed in standard procedures. Expression of FLS2-3xmyc-GFP protein was confirmed by Western blot analysis using anti-myc tag polyclonal antibodies (Upstate) and anti-GFP rabbit serum (Molecular Probes). Plants of the T3 generation were chosen for experiments.

\section{Confocal microscopy}

GFP fluorescence was observed in an Olympus microscope (FV500) after excitation at $488 \mathrm{~nm}$. GFP emission and remaining autofluorescence were detected using the EGFP $(505-525 \mathrm{~nm})$ and PI (>660 nm) filter sets. All samples were imaged with the $60 \times$ oil objective. Pictures were taken in Kahlman frame giving an average of four scans.

Ligand and inhibitor applications

Detached leaves of two independent lines (Ws-0/FLS2-GFP line 4 and Ws-0/FLS2-GFP line 7) were either preincubated for 30 min in $\mathrm{H}_{2} \mathrm{O}$ or in $1 \%$ DMSO at room temperature. Flg22, its variants $\mathrm{flg} 22^{\text {A.tum }}$ and flg $22 \Delta 2$, and elf 26 were added to a final concentration of $10 \mu \mathrm{M}$. Cotreatments with K-252a were done at a final concentration of $10 \mu \mathrm{M}$. The following inhibitors were used: $100 \mu \mathrm{g} / \mathrm{mL}$ BFA, $33 \mu \mathrm{M}$ Wortmannin, 20 $\mu \mathrm{M}$ Oryzalin, $20 \mu \mathrm{M}$ Latrunculin B, and $50 \mu \mathrm{M}$ MG132. All were added $30 \mathrm{~min}$ ahead of flg22 application.

Site-directed mutagenesis

For each construct, FLS2-3xmyc was PCR-amplified and replaced into the StuI and SalI sites of the original FLS2p::FLS2-3xmyc-GFP construct. Amino acid substitutions of T867V, S878A, T1040A, T1072A, and S1118A were achieved by the megaprimer method. Transgenic plants of the T2 generation were chosen for experiments. Binding studies with radiolabeled ${ }^{125} \mathrm{I}$-Tyr-flg22, seedling growth inhibition studies, ROS assays, and bacterial infections were performed as described previously (Zipfel et al. 2004).

\section{Acknowledgments}

We thank B. Hohn, F. Meins, and H. Kohler (all FMI, Basel) for continuous support and technical help, and G. Felix and M. Heinlein for discussions. This work was supported by a grant of the Swiss National Foundation. 


\section{References}

Asai, T., Tena, G., Plotnikova, J., Willmann, M.R., Chiu, W.L., GomezGomez, L., Boller, T., Ausubel, F.M., and Sheen, J. 2002. MAP kinase signalling cascade in Arabidopsis innate immunity. Nature 415: 977 983.

Bauer, Z., Gomez-Gomez, L., Boller, T., and Felix, G. 2001. Sensitivity of different ecotypes and mutants of Arabidopsis thaliana toward the bacterial elicitor flagellin correlates with the presence of receptorbinding sites. J. Biol. Chem. 267: 45669-45676.

Becraft, P.W. 2002. Receptor kinase signaling in plant development. Annu. Rev. Cell Dev. Biol. 18: 163-192.

Chinchilla, D., Bauer, Z., Regenass, M., Boller, T., and Felix, G. 2006. The Arabidopsis receptor kinase FLS2 binds flg22 and determines the specificity of flagellin perception. Plant Cell 18: 1-12.

Chuang, T.H. and Ulevitch, R.J. 2004. Triad3A, an E3 ubiquitin-protein ligase regulating Toll-like receptors. Nat. Immunol. 5: 495-502.

Cook, D.N., Pisetsky, D.S., and Schwartz, D.A. 2004. Toll-like receptors in the pathogenesis of human disease. Nat. Immunol. 5: 975-979.

Emans, N., Zimmermann, S., and Fischer, R. 2002. Uptake of a fluorescent marker in plant cells is sensitive to Brefeldin A and Wortmannin. Plant Cell 14: 71-86.

Engqvist-Goldstein, A.E.Y. and Drubin, D.G. 2003. Actin assembly and endocytosis: From yeast to mammals. Annu. Rev. Cell Dev. Biol. 19: 287-332.

Felix, G., Grosskopf, D.G., Regenass, M., and Boller, T. 1991. Rapid changes of protein phosphorylation are involved in transduction of the elicitor signal in plant cells. Proc. Natl. Acad. Sci. 88: 8831-8834.

Felix, G., Duran, J.D., Volko, S., and Boller, T. 1999. Plants have a sensitive perception system for the most conserved domain of bacterial flagellin. Plant J. 18: 265-276.

Geldner, N., Anders, N., Wolters, H., Keicher, J., Kornberger, W., Muller, P., Delbarre, A., Ueda, T., Nakano, A., and Jurgens, G. 2003. The Arabidopsis GNOM ARF-GEF mediates endosomal recycling, auxin transport, and auxin-dependent plant growth. Cell 112: 219-230.

Gifford, M.L., Robertson, F.C., Soares, D.C., and Ingram, G.C. 2005. Ara bidopsis CRINCLY4 function, internalization, and turnover are dependent on the extracellular crinkly repeat domain. Plant Cell 17: 1154-1166.

Gomez-Gomez, L., Bauer, Z., and Boller, T. 2001. Both the extracellular leucine-rich repeat domain and the kinase activity of FSL2 are required for flagellin binding and signaling in Arabidopsis. Plant Cell 13: $1155-1163$.

Gordon, S. 2002. Pattern recognition receptors: Doubling up for the innate immune response. Cell 111: 927-930.

Haglund, K., Di Fiore, P.P., and Dikic, I. 2003. Distinct monoubiquitin signals in recptor endocytosis. Trends Biochem. Sci. 28: 598-603.

Hammond, D.E., Urbe, S., Vande Woude, G.F., and Clague, M.J. 2001 Down-regulation of Met, the receptor for hepatocyte growth factor. Oncogene 20: 2761-2770.

Hubbard, S.R. and Till, J.H. 2000. Protein tyrosine kinase structure and function. Annu. Rev. Biochem. 69: 373-398.

Katzmann, D.J., Odorizzi, G., and Emr, S.D. 2002. Receptor downregulation and multivesicular-body sorting. Nat. Rev. Mol. Cell Biol. 3: 893-905.

Kim, M.G., da Cunha, L., McFall, A.J., Belkhadir, Y., DebRoy, S., Dangl, J.L., and Mackey, D. 2005. Two Pseudomonas syringae Type III effectors inhibit RIN4-regulated basal defense in Arabidopsis. Cell 121: 749-759.

Kunze, G., Zipfel, C., Robatzek, S., Niehaus, K., Boller, T., and Felix, G. 2004. The $\mathrm{N}$ terminus of bacterial elongation factor Tu elicits innate immunity in Arabidopsis plants. Plant Cell 16: 3496-3507.

Kurten, R.C. 2003. Sorting mortifs in receptor trafficking. Adv. Drug Deliv. Rev. 55: 1405-1419.

Liew, F.Y., Xu, D., Brint, E.K., and O'Neill, L.A. 2005. Negative regulation of toll-like receptor-mediated immune responses. Nat. Rev. Immunol. 5: 446-458.

Longva, K.E., Blystad, F.D., Stang, E., Larsen, A.M., Johannessen, L.E., and Madshus, I.H. 2002. Ubiquitination and proteasomal activity is required for transport of the EGF receptor to inner membranes of multivesicular bodies. J. Cell Biol. 156: 843-854.

Mathur, J. and Chua, N.H. 2000. Microtubule stabilization leads to growth reorientation in Arabidopsis trichomes. Plant Cell 12: 465
477.

Meckel, T., Hurst, A.C., Thiel, G., and Homann, U. 2004. Endocytosis against high turgor: Intact guard cells of Vicia faba constitutively endocytose fluorescently labelled plasma membrane and GFP-tagged K+-channel KAT1. Plant J. 39: 182-193.

Nasrallah, J.B. 2000. Cell-cell signalling in the self-incompatibility response. Curr. Opin. Plant Biol. 3: 368-373.

Nuhse, T.S., Stensballe, A., Jensen, O.N., and Peck, S.C. 2004. Phosphoproteomics of the Arabidopsis plasma membrane and a new phosphorylation site database. Plant Cell 16: 2394-2405.

Paciorek, T., Zazimalova, E., Ruthardt, N., Petrasek, J., Stierhof, Y.D. Kleine-Vehn, J., Morris, D.A., Emans, N., Jurgens, G., Geldner, N., et al. 2005. Auxin inhibits endocytosis and promotes its own efflux from cells. Nature 435: 1251-1256.

Pasare, C. and Medzhitov, R. 2004. Toll-like receptors: Linking innate and adaptive immunity. Microbes Infect. 6: 1382-1387.

Ron, M. and Avni, A. 2004. The receptor for the fungal elicitor ethyleneinducing xylanase is a member of a resistance-like gene family in tomato. Plant Cell 16: 1604-1615.

Russinova, E., Borst, J.W., Kwaitaal, M., Cano-Delgado, A., Yin, Y., Chory, J., and de Vries, S.C. 2004. Heterodimerization and endocytosis of Arabidopsis brassinosteroid receptors BRI1 and AtSERK3 (BAK1). Plant Cell 16: 3216-3229.

Samaj, J., Baluska, F., Voigt, B., Schlicht, M., Volkmann, D., and Menzel, D. 2004. Endocytosis, actin cytoskeleton, and signaling. Plant Physiol. 135: 1150-1161.

Shah, K., Russinova, E., Gadella Jr., T.W., Willemse, J., and de Vries, S.C. 2002. The Arabidopsis kinase-associated protein phosphatase controls internalization of the somatic embryogenesis receptor kinase 1. Genes \& Dev. 16: 1707-1720.

Shiu, S.H. and Bleecker, A.B. 2001. Receptor-like kinases from Arabidopsis form a monophyletic gene family related to animal receptor kinases. Proc. Natl. Acad. Sci. 98: 10763-10768.

Smith, K.D., Andersen-Nissen, E., Hayashi, F., Strobe, K., Bergman, M.A., Barrett, S.L., Cookson, B.T., and Aderem, A. 2003. Toll-like receptor 5 recognizes a conserved site on flagellin required for protofilament formation and bacterial motility. Nat. Immunol. 4: 12471253.

Sorkin, A. and Von Zastrow, M. 2002. Signal transduction and endocytosis: Close encounters of many kinds. Nat. Rev. Mol. Cell Biol. 3 600-614.

Tse, Y.C., Mo, B., Hillmer, S., Zhao, M., Lo, S.W., Robinson, D.G., and Jiang, L. 2004. Identification of multivesicular bodies as prevacuolar compartments in Nicotiana tabacum BY-2 cells. Plant Cell 16: 672693.

Underhill, D.M. and Ganter, B. 2004. Integration of Toll-like receptor and phagocytic signaling for tailored immunity. Microbes Infect. 6: 13681373.

van Kerkhof, P. and Strous, G.J. 2001. The ubiquitin-proteasome pathway regulates lysosomal degradation of the growth hormone receptor and its ligand. Biochem. Soc. Trans. 29: 488-493.

Wang, Z.Y., Seto, H., Fujioka, S., Yoshida, S., and Chory, J. 2001. BRI1 is a critical component of a plasma membrane receptor for plant steroids. Nature 410: 380-383.

Wang, X., Goshe, M.B., Soderblom, E.J., Phinney, B.S., Kuchar, J.A., Li, J., Asami, T., Yoshida, S., Huber, S.C., and Clouse, S.D. 2005. Identification and functional analysis of in vivo phosphorylation sites of the Arabidopsis Brassinosteroid-Insensitive1 receptor kinase. Plant Cell 17: 1685-1703.

Zipfel, C., Robatzek, S., Navarro, L., Oakeley, E., Jones, J.D.G., Felix, G., and Boller, T. 2004. Bacterial disease resistance in Arabidopsis through flagellin perception. Nature 428: 764-767. 


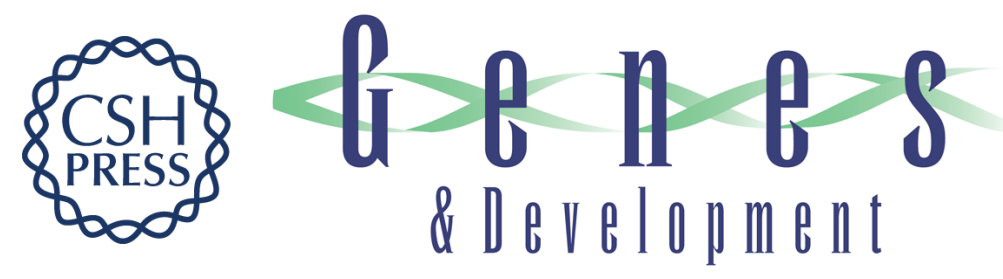

\section{Ligand-induced endocytosis of the pattern recognition receptor FLS2 in Arabidopsis}

Silke Robatzek, Delphine Chinchilla and Thomas Boller

Genes Dev. 2006, 20:

Access the most recent version at doi:10.1101/gad.366506

\section{Supplemental http://genesdev.cshlp.org/content/suppl/2006/02/15/20.5.537.DC1 Material}

References This article cites 42 articles, 15 of which can be accessed free at: http://genesdev.cshlp.org/content/20/5/537.full.html\#ref-list-1

\section{License}

Email Alerting

Receive free email alerts when new articles cite this article - sign up in the box at the top Service 